Allergy News

\section{Bewegte Immunzellen}

rei amerikanische Arbeitsgruppen konnten mit Hilfe der Zwei-Photonen-Laser-Rastermikroskopie erstmals die Bewegungen von Immunzellen per Video dokumentieren: Sabine Stoll vom National Institute of Allergy and Infectious Diseases beobachtete zusammen mit ihren Kollegen, wie T-Zellen mit dendritischen Zellen interagieren. Die Arbeitsgruppe um Philippe Bousso von der University of California in Berkely verfolgte live die zellulären Kontakte bei der Reifung von T-Zellen im Thymus. Mark Miller und seine Kollegen von der University of California in Irvine verglichen in vivo die Be-

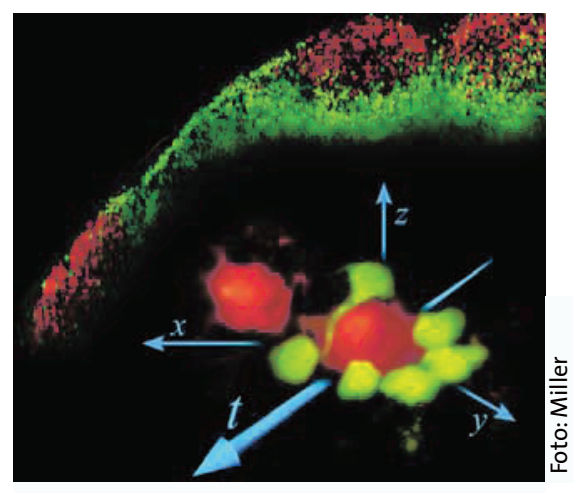

wegungen von T- und B-Zellen im murinen Lymphknoten.

es

Miller MJ et al. Science 2002; 296:

1869-73;

Stoll S et al. Science 2002; 296: 1873-6;

Bousso $P$ et al. Science 2002; 296:

$1876-80$

\title{
Hitzebeständige Sellerie-Allergene
}

D atienten mit einer Sellerieallergie müssen auf das schmackhafte Doldengewächs nicht nur im rohen, sondern auch im gekochten Zustand ver-

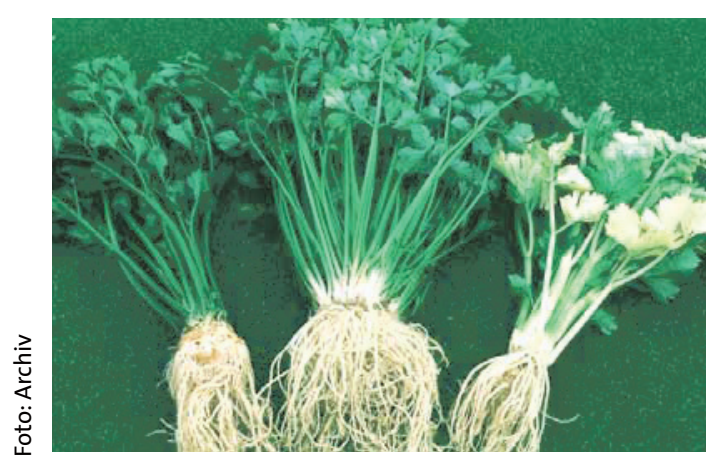

zichten. Die im Sellerie enthaltenen Allergene sind nämlich, wie ein europäisches Wissenschaftler-Team herausgefunden hat, gegen hohe Temperaturen äußerst resistent. Die getesteten Probanden reagierten sogar auf Sellerie allergisch, der bei $100{ }^{\circ} \mathrm{C}$ bis zu 76 Minuten lang gekocht worden war. An dem doppelblinden, plazebokontrollierten Experiment nahmen insgesamt zwölf Patienten mit einer Sellerieallergie teil.

mwe

Ballmer-Weber BK et. al. Allergy 2002; 57: 228-35

\section{Feuchte Wohnungen fördern Allergien}

\footnotetext{
- ine finnische Arbeitsgruppe unter- suchte, ob Schimmel und Feuchtigkeit in Wohnungen Einfluss auf die Häufigkeit des Auftretens von allergischer Rhinitis bzw. Konjunktivitis, Asthma bronchiale und atopischem Ekzem haben. Dazu werteten sie Fragebögen aus, die an über 10.000 finnische Studenten verschickt worden waren. Statistisch signifikant häufig hatten Studenten aus feuchten bzw. schimmeligen Wohnungen Asthma. Auch litten
}

Studenten mit atopischer Anamnese in solchen Wohnungen häufiger an allergischer Rhinitis und atopischem Ekzem als die in trockenen Behausungen lebenden Kommilitonen. Die Autoren machen überwiegend die vermehrte Schimmelbildung und den erhöhten Milbenbestand in feuchten Wohnungen für diese Befunde verantwortlich.

tp

Kilpelainen $\mathbf{M}$ et al. Thorax 2001; 56 : 462-7

\section{Schützen Allergien vor Diabetes?}

topiker scheinen seltener an Typ-1Diabetes zu erkranken. Diese Beobachtung machten Mediziner der Universität Helsinki, die die Häufigkeit von Asthma und Allergien bei Kindern mit Diabetes mellitus juvenilis untersuchten. Beim Vergleich von 306 jungen Diabetikern mit 406 gleichaltrigen Kontrollkindern ergab sich eine inverse Beziehung zwischen der Erkrankungshäufigkeit für Diabetes und der für Asthma (Odds Ratio 0,49) oder einer Tierhaarallergie $(0,67)$. Weniger stark ausgeprägt war der Zusammenhang bei einer Pollenallergie. Faktoren, die eine atopische Erkrankung begünstigen, könnten, so die gewagte These der Finnen, somit vor Typ-1-Diabetes schützen.

Mattila PS et al. Diabetes Care 2002; 25: 865-8

\section{Katzenhaarallergiker arbeitsunfähig?}

ei einem im Außendienst tätigen Versicherungsagenten bewirkt eine bei ihm vorliegende Katzenhaarallergie im Regelfall keine vollständige Arbeitsunfähigkeit, hat das Oberlandesgericht Köln (Az.: 5 U 22/00) entschieden. Dem Versicherungsnehmer ist es zuzumuten, seine Arbeitstätigkeit so zu legen, dass Hausbesuche bei Katzenhaltern weitestgehend ausgeschlossen sind. Die beklagte Krankentagegeldversicherung muss daher, wegen fehlender Arbeitsunfähigkeit, kein Krankentagegeld bezahlen.

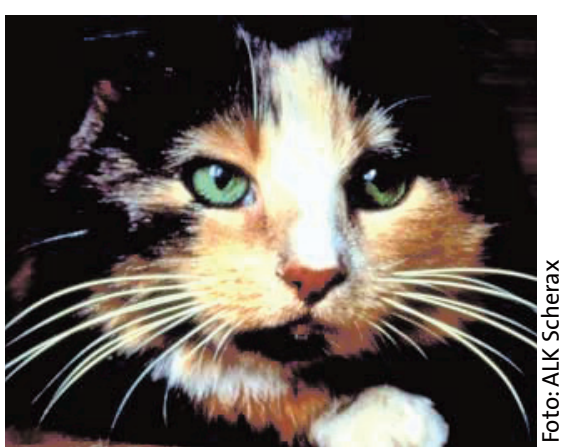

